V. D. Tonchev

Nagoya Math. J.

Vol. 104 (1986), 163-174

\title{
HADAMARD MATRICES OF ORDER 36 WITH AUTOMORPHISMS OF ORDER 17
}

\author{
VLADIMIR D. TONCHEV \\ Dedicated to Professor Noboru Ito on his 60th birthday
}

\section{§1. Introduction}

A Hadamard matrix of order $n$ is an $n$ by $n$ matrix of 1's and - 1's such that $H H^{t}=n I$. In such a matrix $n$ is necessarily 1,2 or a multiple of 4. Two Hadamard matrices $H_{1}$ and $H_{2}$ are called equivalent if there exist monomial matrices $P, Q$ with $P H_{1} Q=H_{2}$. An automorphism of a Hadamard matrix $H$ is an equivalence of the matrix to itself, i.e. a pair $(P, Q)$ of monomial matrices such that $P H Q=H$. In other words, an automorphism of $H$ is a permutation of its rows followed by multiplication of some rows by -1 , which leads to reordering of its columns and multiplication of some columns by -1 . The set of all automorphisms form a group under composition called the automorphism group (Aut $H$ ) of $H$. For a detailed study of the basic properties and applications of Hadamard matrices see, e.g. [1], [7, Chap. 14], [8].

The equivalence classes of Hadamard matrices of orders not exceeding 20 has been determined by Hall [5], [6]. More recently, Ito et al. [11] completed the classification of the Hadamard matrices of order 24. For the next order 28, it is known that the only primes which can divide the group order are 13, 7, 3 and 2, and the matrices possessing automorphisms of order 13 or 7 are found [17], [18]. A rough but susceptible lower bound for the number of equivalence classes of Hadamard matrices of order 32 can be obtained from a result of Norman [13], stating that there are at least 1266891 non-isomorphic Hadamard 2-(31, 15,7) designs, and hence at least $1266891 / 32^{2}$ inequivalent Hadamard matrices of order 32.

Hadamard matrices of order 36 have been extensively studied [2], [10], [20]. It was proved by Ito [9] that if a Hadamard matrix $H$ of order $n$ possesses a "known" doubly-transitive automorphism group then either $H$

Received September 9, 1985. 
is of quadratic-residue type, or $n=36$. A Hadamard matrix of order 36 with a doubly-transitive group was recently constructed by Ito and Leon [10].

In this paper we begin a classification of Hadamard matrices of order 36 by means of "local" properties of their automorphism groups, namely, by considering the possible primes dividing the group order. If $p$ is an odd prime dividing the group order of a Hadamard matrix of order $n$ $(n \geqq 4)$, then either $p$ divides $n$ or $n-1$, or $p \leqq n / 2-1$; moreover, if $p$ does not divide $n$, then $p$ must be an order of an automorphism of a symmetric $2-(n-1, n / 2-1, n / 4-1)$ design [17]. In particular, the largest prime which can divide the group order of a Hadamard matrix of order 36 is 17. The Paley matrix [14], [7], which is undoubtedly the first Hadamard matrix of order 36 ever found, admits an automorphism of order 17. It is perhaps worth noting that none of the 80 Hadamard matrices arising from Steiner triple systems of order 15 [2] possesses automorphisms of order 17.

It is our aim in this paper to show that up to equivalence there are precisely 11 Hadamard matrices of order 36 with automorphisms of order 17. From these 11 matrices, only the Paley matrix has a transitive automorphism group (of order $19584=2^{7} \cdot 3^{2} \cdot 17$ ), while the remaining matrices all have full automorphism groups of order 68 . We use the same method as in [17]. It is not difficult to see that if a Hadamard matrix $H$ of order 36 admits an automorphism of order 17, then $H$ is equivalent to a normalized matrix of the form

$$
\left[\begin{array}{rrr}
1 & \cdots & 1 \\
& 1 \\
M & \vdots \\
& & 1
\end{array}\right]
$$

where $M$ is a $(-1,1)$ incidence matrix of a Hadamard 2-(35, 17, 8) design with an automorphism of order 17 . Further, a 2-(35, 17, 8) design with an automorphism of order 17 has a block, the derived design with respect to which is a cyclic $2-(17,8,7)$ design. Hence one can obtain the Hadamard $2-(35,17,8)$ designs with automorphisms of order 17 as embeddings of cyclic $2-(17,8,7)$ designs. The isomorphism classes of cyclic $2-(17,8,7)$ designs are described in [19]. A table with their representatives is given in Section 2. In Section 3 we study the Hadamard $2-(35,17,8)$ and $3-(36,18,8)$ designs with automorphisms of order 17. There are precisely 21 isomorphism classes of $2-(35,17,8)$ designs, and 11 classes of $3-(36,18,8)$ designs 
with automorphisms of order 17. In the last Section 4 we show that the 11 Hadamard matrices obtained from the non-isomorphic $3-(36,18,8)$ designs are pairwise inequivalent.

\section{§2. Cyclic 2-(17, 8, 7) designs}

Let $X=G F(17)=\{0,1,2, \cdots, 16\}$ be the point set of a $2-(17,8,7)$ design which is invariant under the cyclic group $Z_{17}$, i.e. under the permutation $(0,1,2, \cdots, 16)$. The set of all $\left({ }_{8}^{17}\right)$ 8-element subsets of $X$ is partitioned into 1430 orbits under the action of $Z_{17}$. We checked by computer that exactly 161 pairs of these orbits form a $2-(17,8,7)$ design. The images of the blocks of a cyclic 2-design with point set $G F(p)$ ( $p$ a prime) under an affine transformation of $G F(p)$ form again a cyclic design isomorphic to the initial design, and by a theorem of Bays-Lambossy (cf. [3, p. 225]) the converse is also true: two cyclic designs on a prime number of points are isomorphic if and only if they are affine equivalent. The set of the 161 cyclic $2-(17,8,7)$ designs is thus divided into 11 isomorphism classes: 10 classes consisting of 16 designs each, and one class of a single design. Representatives for these designs are listed in Table 1.

Table 1. Cyclic 2-(17,8,7) designs

\begin{tabular}{rllc} 
No. & \multicolumn{2}{c}{ Representatives of block orbits } & Group order \\
\hline 1 & $(1,2,3,4,5,7,11,14)$, & $(1,2,3,5,8,10,13,14)$ & 17 \\
2 & $(1,2,3,4,5,7,11,14)$, & $(1,2,3,7,8,11,13,16)$ & 17 \\
3 & $(1,2,3,4,5,8,9,13)$, & $(1,2,4,6,9,10,12,16)$ & 17 \\
4 & $(1,2,3,4,5,8,9,13)$, & $(1,2,4,8,10,11,14,16)$ & 17 \\
5 & $(1,2,3,4,5,8,10,14)$, & $(1,2,3,6,8,9,11,15)$ & 17 \\
6 & $(1,2,3,4,5,8,10,14)$, & $(1,2,3,6,10,12,13,15)$ & 17 \\
7 & $(1,2,3,4,6,7,11,13)$, & $(1,2,3,5,6,9,12,14)$ & 17 \\
8 & $(1,2,3,4,6,7,12,14)$, & $(1,2,4,5,8,9,11,13)$ & 17 \\
9 & $(1,2,3,4,6,9,11,15)$, & $(1,2,3,5,6,11,12,16)$ & 17 \\
10 & $(1,2,3,4,6,9,11,15)$, & $(1,2,3,5,9,10,15,16)$ & $2^{4} .17$ \\
11 & $(1,2,3,5,11,13,14,15),(1,2,4,5,7,11,12,16)$ &
\end{tabular}

The full automorphism groups of these designs can be found with the help of Sims' table of primitive permutation groups of degree 17 [15], or by computer using an algorithm of Gibbons [4]. The design 11 has the affine group of $G F(17)$ as a full automorphism group, while the groups of the remaining designs are all of order 17. For more details see [19]. 


\section{$\S 3$. Hadamard $2-(35,17,8)$ and $3-(36,18,8)$ designs with automorphisms of order 17}

Let $D$ be a symmetric $2-(35,17,8)$ design with point set $\{1,2, \cdots, 35\}$, and an automorphism $\beta$ of order 17. We can assume that $\beta=(1,2, \cdots, 17)$ (18) $(19,20, \cdots, 35)$, and the blocks are labeled in such a way that the block fixed by $\beta$ is the last one and consists of the points $1,2, \cdots, 17$, and the fixed point 18 occurs in the first 17 blocks. Then $D$ has an incidence matrix of the form

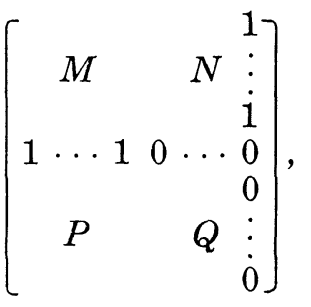

where $M, M, P, Q$ are circulant matrices,

$$
(M, N)
$$

is an incidence matrix of a cyclic $2-(17,8,7)$ design, and

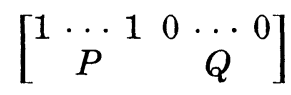

is an incidence matrix of a $2-(18,9,8)$ design with an automorphism of order 17. Similarly,

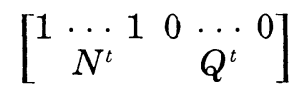

is an incidence matrix of a $2-(18,9,8)$ design with an automorphism of order 17 , and

$$
\left(M^{t}, P^{t}\right)
$$

is an incidence matrix of a cyclic $2-(17,8,7)$ design. Having a quadruple of designs with incidence matrices (2)-(5), we can obtain a symmetric design (if one exist) by fixing $M, N, P$ and permuting the rows of $Q$ cyclically while the matrix (1) produces a design. Let us note that the $2-(18,9,8)$ design with an automorphism of order 17 are easily derived from the cyclic $2-(17,8,7)$ designs: if $(2)$ is an incidence matrix of a cyclic $2-(17,8,7)$ design, then 


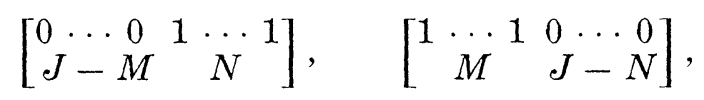

where $J$ is the all-one matrix, are both incidence matrices of $2-(18,9,8)$ designs with an automorphism of order 17. Conversely, if (3) is an incidence matrix of a $2-(18,9,8)$ design with an automorphism of order 17 , then $(J-P, Q)$ is an incidence matrix of a cyclic $2-(17,8,7)$ design.

Another more general way to embed the cycilc $2-(17,8,7)$ designs into symmetric $2-(35,17,8)$ designs is to use the algorithm from [16], based on the observation that if a part (i.e. several rows) of the incidence matrix of a $2-(v, k, \lambda)$ design is given, then any missing row lies in the orthogonal complement of the vector space over $G F(p)$ generated by the given rows, where $p$ is a prime dividing $\lambda$.

Let us remark that if (3) is an incidence matrix of a $2-(18,9,8)$ design completing the design (2) to a symmetric design (1), then the complementary design of (3), i.e. the design with incidence matrix

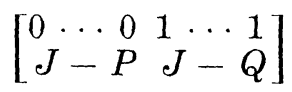

also completes the design (2) to a symmetric design. Therefore, if a $2-(17,8,7)$ design admits an embedding it admits at least two (not necessarily nonisomorphic). It turns out that each of the 11 cyclic $2-(17,8,7)$ designs is embeddable in exactly two symmetric designs. Since the design 11 admits an automorphism of order 2 interchanging the two cyclic block orbits, the symmetric designs thus obtained are given in Table 2, where in all cases a block $B_{35}=\{1,2, \cdots, 17\}$ should be added.

Table 2. Hadamard 2-(35, 17, 8) designs with automorphisms of 17

\begin{tabular}{cl} 
Design & \multicolumn{1}{c}{ Base blocks } \\
\hline$D_{1 A}$ & $B_{1}=(1,2,3,4,5,7,11,14,18,20,22,23,24,28,29,32,34)$ \\
& $B_{18}=(1,2,3,5,8,10,13,14,19,25,26,27,29,30,32,33,34)$ \\
$D_{1 B}$ & $B_{1}=(1,2,3,4,5,7,11,14,19,21,25,26,27,30,31,33,35)$ \\
& $B_{18}=(1,2,3,5,8,10,13,14,18,20,21,22,23,24,28,31,35)$ \\
$D_{2 A}$ & $B_{1}=(1,2,3,4,5,7,11,14,18,22,23,24,26,29,31,34,35)$ \\
& $B_{18}=(1,2,3,7,8,11,13,16,19,25,26,27,29,30,32,33,34)$ \\
$D_{2 B}$ & $B_{1}=(1,2,3,4,5,7,11,14,19,20,21,25,27,28,30,32,33)$ \\
& $B_{18}=(1,2,3,7,8,11,13,16,18,20,21,22,23,24,28,31,35)$ \\
$D_{3 A}$ & $B_{1}=(1,2,3,4,5,8,9,13,18,19,21,23,24,26,30,32,33)$ \\
& $B_{18}=(1,2,4,6,9,10,12,16,19,25,26,27,28,30,31,32,35)$ \\
$D_{3 B}$ & $B_{1}=(1,2,3,4,5,8,9,13,20,22,25,27,28,29,31,34,35)$ \\
& $B_{18}=(1,2,4,6,9,10,12,16,18,20,21,22,23,24,29,33,34)$
\end{tabular}


Table 2. (Continued)

\begin{tabular}{|c|c|}
\hline Design & Base blocks \\
\hline$D_{4 A}$ & $\begin{array}{l}B_{1}=(1,2,3,4,5,8,9,13,18,21,23,24,26,28,31,32,34) \\
B_{18}=(1,2,4,8,10,11,14,16,19,25,26,27,28,30,31,32,35)\end{array}$ \\
\hline$D_{4 B}$ & $\begin{array}{l}B_{1}=(1,2,3,4,5,8,9,13,19,20,22,25,27,29,30,33,35) \\
B_{18}=(1,2,4,8,10,11,14,16,18,20,21,22,23,24,29,33,34)\end{array}$ \\
\hline$D_{5 A}$ & $\begin{array}{l}B_{1}=(1,2,3,4,5,8,10,14,18,19,22,23,24,27,31,33,34) \\
B_{18}=(1,2,3,6,8,9,11,15,19,25,26,27,29,30,31,33,35)\end{array}$ \\
\hline$D_{5 B}$ & $\begin{array}{l}B_{1}=(1,2,3,4,5,8,10,14,20,21,25,26,28,29,30,32,35) \\
B_{18}=(1,2,3,6,8,9,11,15,18,20,21,22,23,24,28,32,34)\end{array}$ \\
\hline$D_{6 A}$ & $\begin{array}{l}B_{1}=(1,2,3,4,5,8,10,14,18,19,22,23,24,27,29,30,32) \\
B_{18}=(1,2,3,6,10,12,13,15,19,25,26,27,29,30,31,33,35)\end{array}$ \\
\hline$D_{6 B}$ & $\begin{array}{l}B_{1}=(1,2,3,4,5,8,10,14,20,21,25,26,28,31,33,34,35) \\
B_{18}=(1,2,3,6,10,12,13,15,18,20,21,22,23,24,28,32,34)\end{array}$ \\
\hline$D_{7 A}$ & $\begin{array}{l}B_{1}=(1,2,3,4,6,7,11,13,18,19,21,22,23,27,29,32,35) \\
B_{18}=(1,2,3,5,6,9,12,14,19,24,25,26,27,29,31,32,33)\end{array}$ \\
\hline$D_{7 B}$ & $\begin{array}{l}B_{1}=(1,2,3,4,6,7,11,13,20,24,25,26,28,30,31,33,34) \\
B_{18}=(1,2,3,5,6,9,12,14,18,20,21,22,23,28,30,34,35)\end{array}$ \\
\hline$D_{\varepsilon A}$ & $\begin{array}{l}B_{1}=(1,2,3,4,6,7,12,14,18,20,22,23,26,27,29,30,35) \\
B_{18}=(1,2,4,5,8,9,11,13,20,21,22,23,26,31,32,33,35)\end{array}$ \\
\hline$D_{8 B}$ & $\begin{array}{l}B_{1}=(1,2,3,4,6,7,12,14,19,21,24,25,28,31,32,33,34) \\
B_{18}=(1,2,4,5,8,9,11,13,18,19,24,25,27,28,29,30,34)\end{array}$ \\
\hline$D_{9 . A}$ & $\begin{array}{l}B_{1}=(1,2,3,4,6,9,11,15,18,22,23,28,29,31,32,33,35) \\
B_{18}=(1,2,3,5,6,11,12,16,20,21,22,24,26,27,29,34,35)\end{array}$ \\
\hline$D_{9 B}$ & $\begin{array}{l}B_{1}=(1,2,3,4,6,9,11,15,19,20,21,24,25,26,27,30,34) \\
B_{18}=(1,2,3,5,6,11,12,16,18,19,23,25,28,30,31,32,33)\end{array}$ \\
\hline$D_{10 . A}$ & $\begin{array}{l}B_{1}=(1,2,3,4,6,9,11,15,18,19,24,25,29,31,32,33,35) \\
B_{18}=(1,2,3,5,9,10,15,16,20,21,22,24,26,27,29,34,35)\end{array}$ \\
\hline$D_{10 B}$ & $\begin{array}{l}B_{1}=(1,2,3,4,6,9,11,15,20,21,22,23,26,27,28,30,34) \\
B_{18}=(1,2,3,5,9,10,15,16,18,19,23,25,28,30,31,32,33)\end{array}$ \\
\hline$D_{11 A}$ & $\begin{array}{l}B_{1}=(1,2,3,5,11,13,14,15,18,19,21,22,24,28,29,33,35) \\
B_{18}=(1,2,4,5,7,11,12,16,19,23,24,28,30,31,32,33,34)\end{array}$ \\
\hline
\end{tabular}

In order to establish the nonisomorphism of the designs from Table 2, we count the number $m_{i}$ of pairs of points occuring together with a given point in precisely $i$ blocks $(0 \leqq i \leqq 8)$. Of course, it is sufficient to do this only for a triple of points belonging to different $\beta$-orbits. The results are given in Table 3 .

Table 3.

\begin{tabular}{|c|c|c|c|c|c|c|c|}
\hline Design & & $\left(m_{0}, m_{1}, \cdots, m_{6}\right)$ & & $\begin{array}{l}\text { Number } \\
\text { of points }\end{array}$ & & Dual design & \\
\hline \multirow[t]{3}{*}{$\bar{D}_{1 . A}$} & 0 & 03420425568 & 0 & 1 & 0 & 03420425568 & 0 \\
\hline & 0 & $\begin{array}{lllll}0 & 31 & 213 & 246 & 71\end{array}$ & 0 & 17 & 0 & 03121324671 & 0 \\
\hline & 0 & $\begin{array}{lllll}0 & 33 & 207 & 252 & 69\end{array}$ & 0 & 17 & 0 & $\begin{array}{llll}0 & 33207 & 252 & 69\end{array}$ & 0 \\
\hline
\end{tabular}


Table 3. (Continued)

\begin{tabular}{|c|c|c|c|c|c|c|c|}
\hline \multirow{2}{*}{$\frac{\text { Design }}{D_{1 B}}$} & \multicolumn{3}{|c|}{$\left(m_{0}, m_{1}, \cdots, m_{6}\right)$} & \multirow{2}{*}{$\begin{array}{l}\text { Number } \\
\text { of points } \\
1\end{array}$} & \multicolumn{3}{|c|}{ Dual design } \\
\hline & 0 & 03420425568 & 0 & & 0 & 03420425568 & 0 \\
\hline & 0 & 03320725269 & 0 & 17 & 0 & 03320725269 & 0 \\
\hline & 0 & 03719526465 & 0 & 17 & 0 & 03719526465 & 0 \\
\hline$D_{2 A}$ & 0 & 03420425568 & 0 & 1 & 0 & 03420425568 & 0 \\
\hline & 0 & 03121324671 & 0 & 17 & 0 & 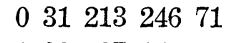 & 0 \\
\hline & 0 & 03320725269 & 0 & 17 & 0 & 03320725269 & 0 \\
\hline$D_{2 B}$ & 0 & 03420425568 & 0 & 1 & 0 & 03420425568 & 0 \\
\hline & 0 & 03320725269 & 0 & 17 & 0 & 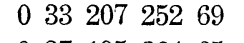 & 0 \\
\hline & 0 & 03719526465 & 0 & 17 & 0 & 03719526465 & 0 \\
\hline$D_{3 A}$ & & $\begin{array}{lllll}17 & 17 & 153 & 340 & 34\end{array}$ & 0 & 1 & 0 & 03420425568 & 0 \\
\hline & 0 & 12422324860 & 5 & 17 & 0 & 02821824962 & 4 \\
\hline & 0 & 12621825161 & 4 & 17 & 0 & 03021424966 & 2 \\
\hline$D_{3 B}$ & 0 & $17 \quad 17 \quad 153 \quad 340 \quad 34$ & 0 & 1 & 0 & 03420425568 & 0 \\
\hline & 0 & 23518528649 & 4 & 17 & 0 & 23419227162 & 0 \\
\hline & 0 & 33617829151 & 2 & 17 & 0 & 43019227560 & 0 \\
\hline$D_{4 A}$ & 0 & 03420425568 & 0 & 1 & & $17 \quad 17 \quad 153 \quad 340 \quad 34$ & 0 \\
\hline & 0 & 02821824962 & 4 & 17 & 0 & 12422324860 & 5 \\
\hline & 0 & $0 \quad 3021424966$ & 2 & 17 & 0 & 12621825161 & 4 \\
\hline$D_{4 B}$ & 0 & 03420425568 & 0 & 1 & & $1717 \quad 15334034$ & 0 \\
\hline & 0 & 23419227162 & 0 & 17 & 0 & 23518528649 & 4 \\
\hline & 0 & 43019227560 & 0 & 17 & 0 & 33617829151 & 2 \\
\hline$D_{5 A}$ & 0 & 03420425568 & 0 & 1 & 0 & $\begin{array}{lllll}0 & 17 & 238 & 255 & 34\end{array}$ & 17 \\
\hline & 0 & 03420126459 & 3 & 17 & 0 & 03719127653 & 4 \\
\hline & 0 & $\begin{array}{lllll}0 & 39 & 189 & 270 & 63\end{array}$ & 0 & 17 & 0 & 04018527659 & 1 \\
\hline$D_{5 B}$ & 0 & 03420425568 & 0 & 1 & 0 & $\begin{array}{llll}0 & 17238 & 255 & 34\end{array}$ & 17 \\
\hline & 0 & $\begin{array}{lllll}0 & 30 & 213 & 252 & 63\end{array}$ & 3 & 17 & 0 & 03220925267 & 1 \\
\hline & 0 & 03719526465 & 0 & 17 & 0 & 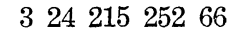 & 1 \\
\hline$D_{6 A}$ & 0 & 01723825534 & 17 & 1 & 0 & 03420425568 & 0 \\
\hline & 0 & 03719127653 & 4 & 17 & 0 & 03420126459 & 3 \\
\hline & 0 & $\begin{array}{lllll}0 & 40 & 185 & 276 & 59\end{array}$ & 1 & 17 & 0 & $\begin{array}{lllll}0 & 39 & 189 & 270 & 63\end{array}$ & 0 \\
\hline$D_{6 B}$ & 0 & 01723825534 & 17 & 1 & 0 & 03420425568 & 0 \\
\hline & 0 & 03220925267 & 1 & 17 & 0 & $\begin{array}{lllll}0 & 30 & 213 & 252 & 63\end{array}$ & 3 \\
\hline & 0 & 32421525266 & 1 & 17 & 0 & 03719526465 & 0 \\
\hline$D_{7 A}$ & 0 & 03418730617 & 17 & 1 & 0 & 03418730617 & 17 \\
\hline & 0 & 03220626158 & 4 & 17 & 0 & 03021225560 & 4 \\
\hline & 0 & 03220925267 & 1 & 17 & 0 & 03420325865 & 1 \\
\hline$D_{7 B}$ & 0 & 03418730617 & 17 & 1 & 0 & 03418730617 & 17 \\
\hline & 0 & 03021225560 & 4 & 17 & 0 & 03220626158 & 4 \\
\hline & 0 & 03420325865 & 1 & 17 & 0 & 03220925267 & 1 \\
\hline$D_{8 A}$ & 0 & 01723825534 & 17 & 1 & 0 & 01723825534 & 17 \\
\hline & 0 & 02223024950 & 10 & 17 & 0 & 02323323467 & 4 \\
\hline & 0 & 03120925859 & 4 & 17 & 0 & 31523624357 & 7 \\
\hline$D_{8 B}$ & 0 & $\begin{array}{lllll}0 & 17 & 238 & 255 & 34\end{array}$ & 17 & 1 & 0 & 01723825534 & 17 \\
\hline & 0 & 02323323467 & 4 & 17 & 0 & 02223024950 & 10 \\
\hline & 0 & 31523624357 & 7 & 17 & 0 & 03120925859 & 4 \\
\hline
\end{tabular}


Table 3. (Continued)

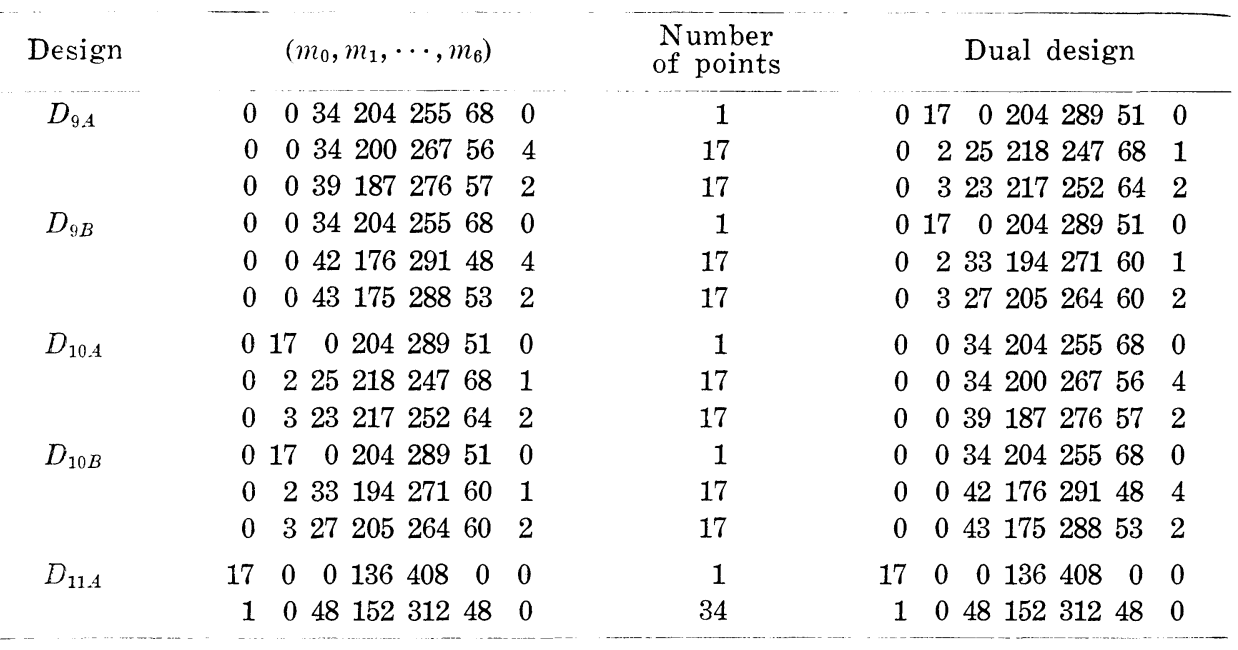

It follows from Table 3 that the designs from Table 2 are all pairwise non-isomorphic with possible exceptions for the pairs $\left(D_{1 A}, D_{2 A}\right)$ and $\left(D_{1 B}, D_{2 B}\right)$. However, $D_{1 A}$ and $D_{2 A}$ (as well as $D_{1 B}$ and $D_{2 B}$ ) can not be isomorphic since an isomorphism must map the fixed block 35 of $D_{1.1}$ onto the block 35 of $D_{2 A}$, and consequently, the cyclic $2-(17,8,7)$ design 1 to the design 2 , which is impossible.

The characteristics $\left(m_{0}, \cdots, m_{6}\right)$ of the dual designs (i.e. those having as incidence matrix the transpose of the matrix of the initial design) show that $D_{11 A}$ is self-dual, and the pairs $\left(D_{3 A}, D_{4 A}\right),\left(D_{3 B}, D_{4 B}\right),\left(D_{5 A}, D_{6 A}\right),\left(D_{5 B}\right.$, $\left.D_{6 B}\right),\left(D_{7 A}, D_{7 B}\right),\left(D_{8 A}, D_{8 B}\right),\left(D_{9 A}, D_{10 A}\right),\left(D_{9 B}, D_{10 B}\right)$ consist of designs which are dual to each other. A comparison of the derived cyclic 2-(17,8,7) designs in the duals of $D_{1 A}, D_{2 A}, D_{1 B}, D_{2 B}$ shows that $\left(D_{1 A}, D_{2 A}\right)$ and $\left(D_{1 B}\right.$, $D_{2 B}$ ) are dual pairs.

The data from Table 3 shows also that all designs but $D_{11 A}$ have full automorphism groups of order 17 . Since each automorphism of $D_{11 A}$ must fix the block 35, the automorphism group of $D_{11 A}$ must be isomorphic to a subgroup of the group of the cyclic $2-(17,8,7)$ design 11 . In fact, the full group of $D_{11 A}$ is of order $8 \cdot 17$, and is generated by $\beta$ and the permutation

$$
\begin{aligned}
c= & (1)(2,3,5,9,17,16,14,10)(4,7,13,8,15,12,6,11) \\
& (18)(19,25,20,27,24,35,23,33)(21,29,28,26,22,31,32,34)(30) .
\end{aligned}
$$

Every Hadamard $2-(4 t+3,2 t+1, t)$ design is extendable in exactly 
one (up to isomorphism) way to a Hadamard $3-(4 t+4,2 t+2, t)$ design by enlarging all blocks with a new point, and adding $4 t+3$ new blocks being the complements of the old blocks. Consequently, two $3-(4 t+4$, $2 t+2, t)$ designs are isomorphic iff they possess a pair of isomorphic derived $2-(4 t+3,2 t+1, t)$ designs. Moreover, the stabilizer of a point in the automorphism group of a $3-(4 t+4,2 t+2, t)$ design $E$ coincides with the automorphism group of the derived $2-(4 t+3,2 t+1, t)$ design with respect to this point, and two points of $E$ are in the same orbit iff the derived designs with respect to these points are isomorphic.

If $E$ is a $3-(36,18,8)$ design being an extension of a $2-(35,17,8)$ design with an automorphism $\beta$ of order 17, then $\beta$ acts on $E$ by fixing the new point. Thus at least two of the derived designs of $E$ have automorphisms of order 17. It is readily seen that a pair of designs $\left(D_{i A}, D_{i B}\right)$ is extended to isomorphic $3-(36,18,8)$ designs, so we have at most 11 non-isomorphic $3-(36,18,8)$ designs with automorphisms of order 17 . We denote the extension of $D_{i A}$ by $E_{i}$. The characteristics $\left(n_{0}, n_{1}, \cdots, n_{7}\right)$ for the extended designs, where $n_{i}$ is the number of triples of points occuring together with a given point in exactly $i$ blocks, are listed in Table 4, and they show that the 11 3-designs are non-isomorphic.

Table 4.

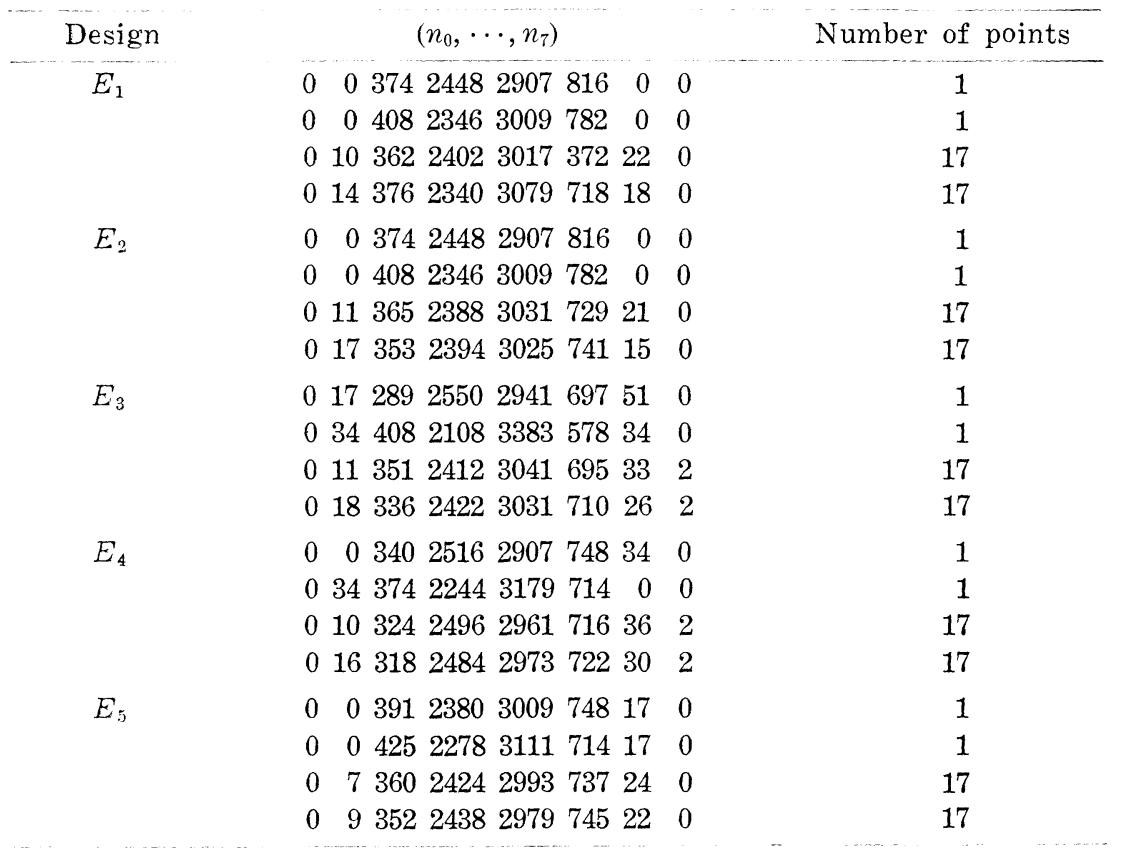


Table 4. (Continued)

\begin{tabular}{|c|c|c|c|}
\hline Design & $\left(n_{0}, \cdots, n_{7}\right)$ & & Number of points \\
\hline$E_{6}$ & $\begin{array}{rrrrrrr}0 & 0 & 442 & 2210 & 3213 & 646 & 34 \\
0 & 17 & 323 & 2482 & 2941 & 765 & 17 \\
0 & 3 & 385 & 2370 & 3045 & 715 & 27 \\
0 & 8 & 382 & 2354 & 3061 & 718 & 22\end{array}$ & $\begin{array}{l}0 \\
0 \\
0 \\
0\end{array}$ & $\begin{array}{r}1 \\
1 \\
17 \\
17\end{array}$ \\
\hline$E_{7}$ & $\begin{array}{lllllll}0 & 0 & 374 & 2414 & 3009 & 714 & 34 \\
0 & 4 & 366 & 2428 & 2975 & 752 & 20 \\
0 & 4 & 378 & 2392 & 3011 & 740 & 20\end{array}$ & $\begin{array}{l}0 \\
0 \\
0\end{array}$ & $\begin{array}{r}2 \\
17 \\
17\end{array}$ \\
\hline$E_{8}$ & $\begin{array}{rrrrrrr}0 & 0 & 306 & 2567 & 2958 & 629 & 85 \\
0 & 17 & 221 & 2737 & 2788 & 714 & 68 \\
0 & 15 & 327 & 2445 & 3046 & 662 & 48 \\
0 & 16 & 314 & 2479 & 3012 & 675 & 47\end{array}$ & $\begin{array}{l}0 \\
0 \\
2 \\
2 \\
2\end{array}$ & $\begin{array}{r}1 \\
1 \\
17 \\
17\end{array}$ \\
\hline$E_{9}$ & $\begin{array}{lllllll}0 & 0 & 425 & 2261 & 3162 & 663 & 34 \\
0 & 0 & 493 & 2057 & 3366 & 595 & 34 \\
0 & 5 & 350 & 2451 & 2990 & 714 & 33 \\
0 & 7 & 376 & 2363 & 3078 & 688 & 31\end{array}$ & $\begin{array}{l}0 \\
0 \\
2 \\
2\end{array}$ & $\begin{array}{r}1 \\
1 \\
17 \\
17\end{array}$ \\
\hline$E_{10}$ & $\begin{array}{lllllll}0 & 34 & 272 & 2533 & 2924 & 765 & 17 \\
0 & 34 & 340 & 2329 & 3128 & 697 & 17 \\
0 & 11 & 343 & 2443 & 2996 & 724 & 26 \\
0 & 13 & 353 & 2403 & 3036 & 714 & 24\end{array}$ & $\begin{array}{l}0 \\
0 \\
2 \\
2\end{array}$ & $\begin{array}{r}1 \\
1 \\
17 \\
17\end{array}$ \\
\hline$E_{11}$ & $\begin{array}{rrrrrrr}17 & 0 & 544 & 1768 & 3672 & 544 & 0 \\
1 & 0 & 352 & 2456 & 2984 & 736 & 0\end{array}$ & $\begin{array}{r}0 \\
16\end{array}$ & $\begin{array}{r}2 \\
34\end{array}$ \\
\hline
\end{tabular}

Since two points lying in the same orbit under the automorphism group must have identical characteristics, it follows from Table 4 and the preceding comments that the only designs which might have automorphism groups larger than $Z_{17}$ are $E_{7}$ and $E_{11}$. But the derived designs of $E_{7}$ with respect to the points having identical characteristics $(0,0,374,2414,3009$, $714,34,0)$ are isomorphic to $D_{7 A}$ and $D_{7 B}$ respectively, which are nonisomorphic. Hence the full group of $E_{7}$ is of order 17 . In the case of $E_{11}$ the derived designs with respect to points 18 and 36 (with characteristics $(17,0,544, \cdots, 0))$ are isomorphic, whence $\mid$ Aut $E_{11}|=2|$ Aut $D_{11 A} \mid=16 \cdot 17$.

\section{$\S 4$. The Hadamard matrices}

Let $M$ be a $(-1,1)$ incidence matrix of a $2-(35,17,8)$ design with an automorphism $\beta$ of order 17. Then bordering $M$ with a column and row of 1's one obtains a Hadamard matrix on which $\beta$ acts by fixing the allone row and column. Two Hadamard matrices obtained from symmetric designs, which are extendable to isomorphic 3-designs are equivalent. More precisely, given a Hadamard matrix $H=\left(h_{i j}\right)$ of order $n=4 t+4$ and given $k(1 \leqq k \leqq n)$, we obtain a 3 - $(4 t+4,2 t+2, t)$ design $E^{k}=E^{k}(H)$ 
with point set $P=\{1,2, \cdots, n\}$ and block set $\left\{B_{1}, \cdots, B_{k-1}, B_{k+1}, \cdots, B_{n}\right.$, $\left.\bar{B}_{1}, \cdots, \bar{B}_{k-1}, \bar{B}_{k+1}, \cdots, \bar{B}_{n}\right\}$ where $B_{j}=\left\{i: h_{i j}=h_{i k}\right\}$ and $\bar{B}_{j}=P-B_{j}$. The designs $E^{k}(H)$ and $E^{m}(H)$ are isomorphic iff columns $k$ and $m$ of $H$ lie in the same orbit of Aut $H$; more generally, $E^{k}\left(H_{1}\right)$ and $E^{k}\left(H_{2}\right)$ are isomorphic iff $H_{1}$ and $H_{2}$ are equivalent under a signed permutation mapping the column $k$ of $H_{1}$ to column $m$ of $H_{2}$. Moreover, the automorphism group of $E^{k}(H)$ acting on points is permutation isomorphic to the stabilizer in Aut $H$ of column $k$, acting on signed rows. The automorphism group of any Hadamard matrix contains a subgroup of order 2 generated by $(-I,-I)$ which fixes all rows and columns. Hence the order of the automorphism group is Aut $H=2 \mid$ Aut $E^{k}(H) \mid l_{k}$, where $l_{k}$ is the length of the orbit of column $k$ under Aut $H$ [12].

The Hadamard matrices obtained from the eleven $2-(35,17,8)$ designs $D_{1 A}, \cdots, D_{11 A}$ can be distinguished by the characteristics $\left(n_{0}, \cdots, n_{7}\right)$ of the related 3-designs $E^{k}$. Evidently in such a matrix the 3-designs obtained with respect to the columns fixed by $\beta$ are isomorphic. The matrix corresponding to $D_{11 A}$, which is easily seen to be equivalent to the Paley matrix, has the property that its 3 -designs $E^{k}(1 \leqq k \leqq 36)$ all have identical characteristic sets, and further analysis shows that all these 3-designs are isomorphic to $E_{11}$. Hence the order of the group of the Paley matrix is $2 \cdot 36 \cdot 272=19584$.

In the remaining 10 matrices, the columns are divided into three orbits; two of length 17 , and one of length 2 . Therefore, all these matrices have groups of order $2 \cdot 2 \cdot 17=68$.

\section{REFERENCES}

[1] Th. Beth, D. Jungnickel, H. Lenz, Design theory, Bibliographisches Institut Mannheim/Wien/Zürich, 1985.

[2] F. C. Bussemaker and J. J. Seidel, Symmetric Hadamard matrices of order 36, Ann. N.Y. Acad. Sci., 175 (1970), 66-79.

[ 3 ] M. J. Colbourn and R. A. Mathon, On cyclic Steiner 2-designs, Ann. Discrete Math., 7 (1980), 215-254.

[4] P. B. Gibbons, Computing techniques for the construction and analysis of block designs, Technical Report, No.92, Univ. of Toronto, 1976.

[5] M. Hall, Jr., Hadamard matrices of order 16, Jet Propulation Laboratory Research Summary No. 36-10, 1 (1961), 21-26.

[6] —- Hadamard matrices of order 20, Jet Propulation Technical Report No. 32$761,1965$.

[ 7 ] - Combinatorial Theory, Gion (Blaisdell), Boston, 1967.

[8] A. Hedayat and W. D. Wallis, Hadamard matrices and their applications, Ann. Statist., 6 (1978), 1184-1238. 
[9 ] N. Ito, Hadamard matrices with "doubly transitive" automorphism groups, Arch. Math., 35 (1980), 100-111.

[10] - and J. S. Leon, An Hadamard matrix of order 36, J. Combin. Theory, A 34 (1983), 244-247.

[11] — J. S. Leon and J. Q. Longyear, Classification of 3- $(24,12,5)$ designs and 24dimensional Hadamard matrices, J. Combin. Theory, A 31 (1981), 66-93.

[12] W. M. Kantor, Automorphism groups of Hadamard matrices, J. Combin. Theory, 6 (1969) , 279-281.

[13] C. W. Norman, Non-isomorphic Hadamard designs, J. Combin. Theory, A 21 (1976), 366-344.

[14] R. E. A. C. Paley, On orthogonal matrices, J. Math. Phys. MIT, 12 (1933), 311-320.

[15] C. C. Sims, Computational methods in the study of permutation groups, in "Computational Problems in Abstract Algebra" (J. Leech, Ed.), pp. 169-184, Pergamon, Elmsford, N.Y., 1970.

[16] V. D. Tonchev, Quasi-residual designs, codes and graphs, Colloq. Math. Soc. Janos Bolyai, 37 (1981), 685-695.

[17] - - Hadamard matrices of order 28 with automorphisms of order 13, J. Combin. Theory, A 35 (1983), 43-57.

[18] —- Hadamard matrices of order 28 with automorphisms of order 7, J. Combin. Theory, Ser. A, 40 (1985), 62-81.

[19] —- R. V. Raev, Cyclic 2- $(17,8,7)$ designs and related doubly-even codes, Compt. rend. Acad. bulg. Sci., 35 (1982), 1367-1370.

[20] W. D. Wallis, Hadamard equivalence, Congressus Numerantium, 28 (1980), 15-25.

Institute of Mathematics

Sofia 1090, P.O. Box 373

Bulgaria 